\title{
An Investigation into the Semantics of English Topological Prepositions
}

John D. Kelleher

Technological University Dublin, john.d.kelleher@tudublin.ie

Colm Sloan

Technological University Dublin, colm.sloan@tudublin.ie

Brian Mac Namee

Technological University Dublin, brian.macnamee@tudublin.ie

Follow this and additional works at: https://arrow.tudublin.ie/scschcomart

Part of the Computer Engineering Commons

\section{Recommended Citation}

John Kelleher, Colm Sloan and Brian Mac Namee, "An Investigation into the Semantics of English Topological Prepositions", Cognitive Processing, Vol. 10(2), pp233-236. Springer, 2009 (2009) doi:10.1007/s10339-009-0289-4

This Article is brought to you for free and open access by the School of Computer Sciences at ARROW@TU Dublin. It has been accepted for inclusion in Articles by an authorized administrator of ARROW@TU Dublin. For more information, please contact arrow.admin@tudublin.ie, aisling.coyne@tudublin.ie,gerard.connolly@tudublin.ie.

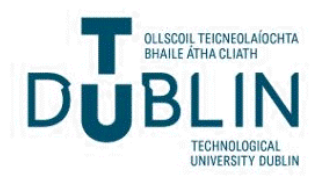




\title{
An Investigation into the Semantics of English Topological Prepositions
}

\author{
John D. Kelleher, Colm Sloan \& Brian Mac Namee \\ John.d.Kelleher@dit.ie|Colm.Sloan@student.dit.ie|Brian.MacNamee@dit.ie \\ DIT AI Group, School of Computing, \\ Dublin Institute of Technology, Dublin, Ireland
}

\begin{abstract}
This paper describes a psycholinguistic experiment that investigates whether the applicability of the topological spatial prepositions "at", "on" or "in" to describe the spatial configuration between two objects is related to the topological relationships between objects being described.
\end{abstract}

Key words: Topological Prepositions, Topological Relations

\section{Introduction}

The topological prepositions "at", "on" and "in" constitute a fundamental set of prepositions in English. The primary constraint on the applicability of these prepositions is the proximity of the object they locate to the landmark being used. This shared constraint often results in an overlap in their range of applicability. Differentiating between the applicability of these prepositions is a problematic issue requiring recourse to both conceptual Herskovits (1986) and functional Conventry \& Garrod (2004) information. However, another factor that may affect the distinctions in their applicability are distinctions in the topological relations that are present in the spatial configurations they are used to describe. By topological relation (as distinct from topological preposition) we mean the type of relations normally used in topography; for example, relations such as inclusion, touching, overlap etc.

The Region Connection Calculus (RCC) Cohn et al. (1997) is a well-known framework that abstractly describes the possible topological relations between two regions. There are several region connection calculi distinguished by the number of topological relationships they identify. The best known of the RCC frameworks is RCC-8. RCC-8 distinguishes 8 topological relations that are possible between two simple regions without holes in 2D space: DISCONNECTED, EXTERNALLY CONNECTED, EQUAL, PARTIALLY OVERLAPPING, TANGENTIAL PROPER PART, TANGENTIAL PROPER PART INVERSE, NON-TANGENTIAL PROPER PART, NON-TANGENTIAL PROPER PART INVERSE.

Moving from 2D to 3D the complexity in formally modeling topological relationships increases dramatically due to the need to represent more complex object types and identifying the relationships between them. For example, the 3D topological framework described in Borrman et al. (2006) uses four types of objects Point, Line Surface and Body. Using these four basic object types the framework distinguishes 9 different types of topological relationship: DISJOINT, EQUAL, CONTAIN, WITHIN, OVERLAP, TOUCH, MEET, ONBOUNDARY, CROSS. 


\section{Methods}

We conducted two experiments that examined how people's judements regarding the appropriateness of the prepositions "at", "on" and "in" to describe the position of one object relative to another object changed as the topological relationships between the two objects changed.

In both experiments a trial consisted of a subject being presented with an image containing two objects and an English sentence of the form The $Y_{1}$ is $X$ the $Y_{2}$. The first $Y$ was was filled with a description of one of the objects, the $X$ was replaced by one of the topological prepositions "at", "on" or "in", and the second blank was filled with a description of the second object. For example: The blue circle is at the green circle. The sentence was presented under the image. The sentence was also spoken by the system at the beginning of each trial. Subjects were instructed that they would be shown sentence-picture pairs and were asked to rate the appropriateness of the sentence to describe the image on a 5-point scale, with one denoting not acceptable at all; three denoting neutral; and five denotes perfectly acceptable. Trials were presented in a random order to control for sequence affects. Trials were self-paced and the experiments lasted about 25 minutes in total. 10 particpants took part in the experiments, 6 male and 4 female.

Experiment 1: The first experiment focused on the relationship between topological prepositions and topological relations in 2D. The images used in this experiment depicted the topological relations distinguished by the RCC-8 model Cohn et al. (1997). The images in this experiment contained two 2D objects (a blue and a green circle) in different topological relationships to each other. The topological relationships illustrated by the $2 \mathrm{D}$ images covered those distinguished by the RCC-8 Cohn et al. (1997) model, with one exception. The EQUAL $E Q\left(R_{1}, R_{2}\right)$ relationship was excluded from the study because it is not possible to graphically represent this relationship in a manner that would allow participants to identify the two objects involved. Note also that although the RCC- 8 distinguishes 8 topological relationships, 2 pairs of these have inverse relationships and so each pair can be represented by 1 image. Consequently, the remaining 7 relationships (i.e. the relations excluding EQUAL) can be graphically represented using 5 images. This resulted in five images being created for this experiment.

In order to control for colour each image was presented in two trials for each preposition in the experiment; in one trial the sentence presented with the image was The blue circle is $X$ the green circle and in the other trial the sentence presented with the image was The green circle is $X$ the blue circle. This resulted in 30 trials: 5 images * 3 prepositions * 2 colour conditions ([blue locatum + green landmark], [green locatum + blue landmark]. .

Experiment 2: The focus of the second experiment was to examine the relationship between topological prepositions and topological relations in 3D. We used the 3D topological famework described in Borrman et al. (2006) as the basis for designing the images for this experiment. Following this framework, the objects used for these images were points, lines, planes and cubes. The framework identifies 9 topological relationships: DISJOINT, EQUAL, TOUCH, MEET, ONBOUNDARY, OVERLAP, CONTAIN, WITHIN, CROSS. Furthermore, following Zlatanova (2000), they identify 69 realisable configurations of these 9 relationships when applied to points, lines, surfaces and cubes. Excluding the configurations identified for the EQUAL 
relation and those configurations that were symmetric within the set defined by a topological relation, 51 configurations remained. Images were created for each of these. As in the first experiment, we controlled for colour by presenting each image twice with the order of the role of each object in relationship reversed; i.e. if an object in an image functioned as the located object in one trial it was used as the landmark in the second trial the image was used in. This resulted in 306 trials: 51 images $* 3$ prepositions $* 2$ colour conditions.

\section{Results}

The results of the 2D experiment are illustrated in Figure 1. Each of the images used in the experiment is shown along with a graph showing the average rating given by subjects for sentences using each of the prepositions covered in the study. From these graphs the following observations can be made regarding the suitability of the three prepositions for each of the topoligical relationships:

DISCONNECTED - All three of the prepositions received very low suitability scores for objects displaying the DISCONNECTED relationship. This suggests that perhaps a proximal term such as near would be more appropriate.

EXTERNALLY CONNECTED - Subjects expressed a preference for "at" in this case and were quite negative about the other two prepositions.

PARTIALLY OVERLAPPING - The responses of subjects were mixed for all three prepositions in this case which suggests that there is some confusion about the exact meaning of this relationship.

TANGENTIAL PROPER PART - A very strong preference for "in" was expressed in this case, with the responses of subjects were mixed with regard to the other two prepositions.

TANGENTIAL PROPER PART INVERSE - While some preference for at was expressed, on also received support. However, it is clear that subjects felt in was entirely inappropriate. It is also important to note here that these responses are almost a direct inversion of those expressed for the non-inverse form of this relationship.

NON-TANGENTIAL PROPER PART - In this case subjects expressed a clear preference for "in".

NON-TANGENTIAL PROPER PART INVERSE - Subjects felt all three of the prepositions were inappropriate for this relationship. It is also interesting to notice that in this case the responses are not a perfect inversion of the non-inverse form of this relationship. 

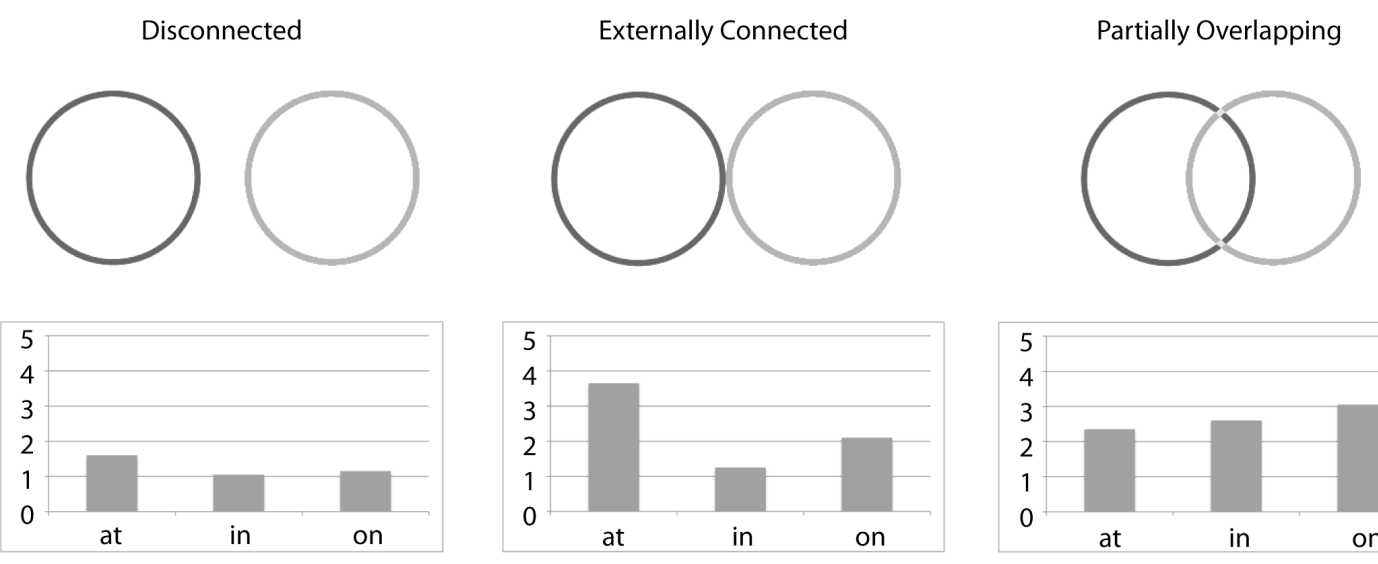

Tangential Proper Part
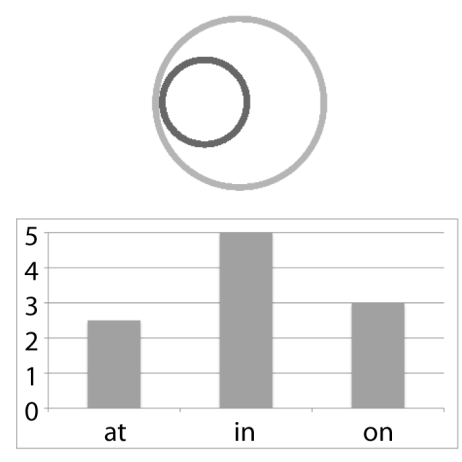

Non-Tangential Proper Part

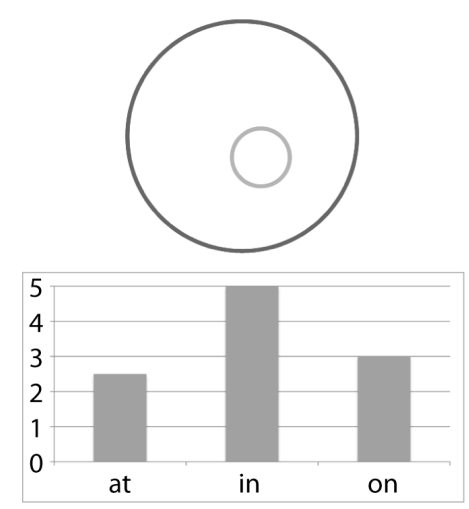

Tangential Proper Part Inverse

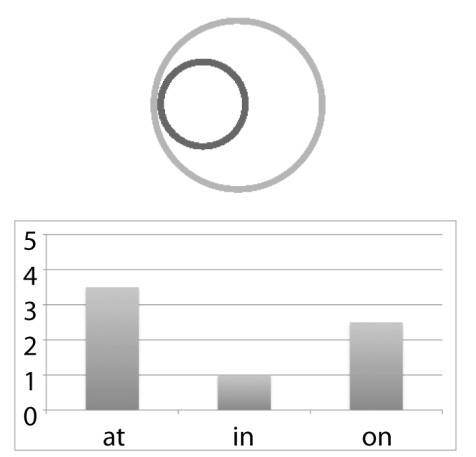

Non-Tangential Proper Part Inverse

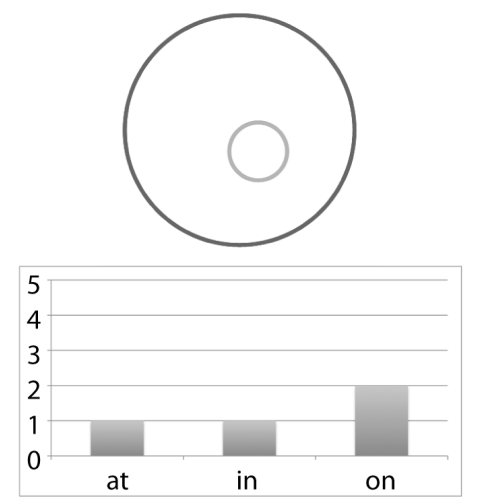

Figure 1: The images used for each of the topological relationships in the 2D experiment. For each relationship a graph of the average response of subjects when asked to rate how well sentences using each of the prepositions "at", "in", and "on" described each image is provided.

The results of the 3D experiment are shown in Table 1 . These results can be summarised as follows:

DISJOINT - Subjects did not find that any of the preopositons were suitable for disjoint realtionships. Again, this suggests the need for a proximal term such as near.

TOUCH - Subjects expressed a clear preference for "at" for images displaying the TOUCH relationship. 
MEET - Similarly to TOUCH, subjects expressed a clear preference for the preposition "at".

ONBOUNDARY - Again in this case "at" was selected as the most appropriate preposition. However, subjects also expressed a strong preference for "on" which is a departure from the MEET and TOUCH relationships.

OVERLAP - Subjects did not express a clear preference for any of the prepositions in this case which suggests that, similarly to the case in the 2D experiment, there is some confusion as to the best way to express this kind of relationship.

CROSS - Like the OVERLAP relationship, there is evidence that some confusion exists as to how best to express this realtionship. Subjects were neutral about which preopsitions were appropriate.

WITHIN - For the WITHIN relationship subjects expressed a clear preference for "in", although it is worth noting that the other two prepositions also received some support.

CONTAIN - While subjects were neutral about "at" and "on" their responses were very clear that "in" was not appropriate for the CONTAIN relationship.

Table 1: The average responses of subjects for each preposition for each relationship in the 3D experiment

\begin{tabular}{|ccc|ccc|ccc|ccc|}
\hline \multicolumn{2}{|c|}{ DISJOINT } & \multicolumn{3}{c|}{ TOUCH } & \multicolumn{3}{c|}{ MEET } & \multicolumn{3}{c|}{ ONBOUNDARY } \\
at in & on & at & in & on & at & in & on & at in & on \\
\hline 2.00 & 1.18 & 2.00 & 3.95 & 1.78 & 2.63 & 4.24 & 2.00 & 2.88 & 4.18 & 2.08 & 3.61 \\
\hline
\end{tabular}

\begin{tabular}{|ccc|ccc|ccc|ccc|}
\hline \multicolumn{2}{|c|}{ OVERLAP } & \multicolumn{3}{c|}{ CROSS } & \multicolumn{3}{c|}{ WITHIN } & \multicolumn{3}{c|}{ CONTAIN } \\
at in & on & at & in & on & at & in & on & at in & on \\
\hline 3.38 & 3.17 & 3.38 & 3.16 & 3.01 & 2.91 & 3.42 & 4.43 & 3.37 & 3.28 & 1.90 & 2.97 \\
\hline
\end{tabular}

While the results of the individual experiments are interesting in their own right it is also interesting that the observations made regarding the $2 \mathrm{D}$ experiment are reinforced by the 3D experiment. In particular the following should be noted:

- For both the 2D DISCONNECTED and the 3D DISJOINT relationships subjects did not feel that any of the prepositions were legitimate descriptions. This suggests that a proximal term such as near is required.

- A preference for "at" emerges in all of those cases in which objects appear to connect: 2D EXTERNALLY CONNECTED and 3D TOUCH, MEET, and ONBOUNDARY. This suggests that connection is an integral part of people's understanding of the preposition "at".

- None of the prepositions are particularly appropriate for overlapping objects. In the 2D PATRIALLY OVERLAPPING and 3D OVERLAP and CROSS cases none of the prepositions considered were found to be suitable.

- The 2D TANGENTIAL PROPER PART and NON-TANGENTIAL PROPER PART relationships correspond with the $3 \mathrm{D}$ WITHIN relationship and a strong preference for "in" is expressed in all of these cases. 
- Similarly, the 2D TANGENTIAL PROPER PART INVERSE and NONTANGENTIAL PROPER PART INVERSE relationships correspond with the 3D CONTAIN relationship and none of the prepositions are suitable in these cases.

\section{Discussion}

In conclusion, the classes of topological relation distinguished by mathematically derived 2D and 3D topologies map to distinctions made by humans when they are interpreting the topological prepositions "at", "on" and "in". One area of research where this finding may have an impact is in the development of natural language generation systems for geographic information systems. The goal of these systems is to generate natural language descriptions of spatial configurations in a spatial database. The findings of this paper point to the fact that the representations used by spatial databases provide a suitable starting point to generate linguistic descriptions using the prepositions "at", "in", and "on".

\section{References}

Borrmann A, van Treeck C, Rank E (2006). Towards a 3D Spatial Query Language for Building Information Models. In: Proc. of the Joint Int. Conf. for Computing and Decision Making in Civil and Building Engineering.

Cohn A, Bennett B, Gooday J, Gotts N (1997) Qualitative Spatial Representation and Reasoning with the Region Connection Calculus. Geoinformatics 1:1-44.

Coventry K and Garrod S (2004) Saying, Seeing and Acting. Pyschology Press, Hove and New York.

Herskovits A (1986) Language and Spatial Cognition: An interdisciplinary study of the prepositions in English. Cambridge University Press, Cambridge.

Zlatanova, S (2000) On 3D Topological Relationships. In: Proc. Of the 11th Internationl Workshop on Database and Expert System applicaitons. Greenwich, London, UK. 